\title{
BMJ Open Effectiveness of interventions to improve hand motor function in individuals with moderate to severe stroke: a systematic review protocol
}

Hewei Wang (D) , ${ }^{1}$ Ray Arceo, ${ }^{2}$ Shugeng Chen, ${ }^{1}$ Li Ding, ${ }^{1}$ Jie Jia (D) , ${ }^{1}$ Jun Yao $^{2}$

To cite: Wang $\mathrm{H}$, Arceo $\mathrm{R}$, Chen S, et al. Effectiveness of interventions to improve hand motor function in individuals with moderate to severe stroke: a systematic review protocol. BMJ Open 2019;9:e032413. doi:10.1136/ bmjopen-2019-032413

- Prepublication history and additional material for this paper are available online. To view these files, please visit the journal online (http://dx.doi org/10.1136/bmjopen-2019032413).

Received 17 June 2019 Revised 13 August 2019 Accepted 02 September 2019

Check for updates

(C) Author(s) (or their employer(s)) 2019. Re-use permitted under CC BY-NC. No commercial re-use. See rights and permissions. Published by BMJ.

${ }^{1}$ Department of Rehabilitation, Huashan Hospital, Fudan University, Shanghai, China ${ }^{2}$ Physical Therapy and Human Movement Sciences, Northwestern University, Chicago, Illinois, USA

Correspondence to

Professor Jun Yao;

j-ya04@northwestern.edu

Dr Jie Jia; shannonjj@126.com

\section{ABSTRACT}

Introduction The human hand is extremely involved in our daily lives. However, the rehabilitation of hand function after stroke can be rather difficult due to the complexity of hand structure and function, as well as neural basis that supports hand function. Specifically, in individuals with moderate to severe impairment following a stroke, previous evidence for effective treatments that recover hand function in this population is limited, and thus has never been reviewed. With the progress of rehabilitation science and tool development, results from more and more clinical trials are now available, thereby justifying conducting a systematic review.

Methods and analysis This systematic review protocol is consistent with the methodology recommended by the Preferred Reporting Items for Systematic Review and Meta-Analysis Protocols and the Cochrane handbook for systematic reviews of interventions. Electronic searches will be carried out in the PubMed, CINAHL, Physiotherapy Evidence Database and Cochrane Library databases, along with manual searches in the reference lists from included studies and published systematic reviews. The date range parameters used in searching all databases is between January 1999 and January 2019. Randomised controlled trials (RCTs) published in English, with the primary outcome focusing on hand motor function, will be included. Two reviewers will screen all retrieved titles, abstracts and full texts, perform the evaluation of the risk bias and extract all data independently. The risk of bias of the included RCTs will be evaluated by the Cochrane Collaboration's tool. A qualitative synthesis will be provided in text and table, to summarise the main results of the selected publications. A meta-analysis will be considered if there is sufficient homogeneity across outcomes. The quality of the included publications will be evaluated by the Grading of Recommendations Assessment, Development and Evaluation system from the Cochrane Handbook for Systematic Reviews of Interventions.

Ethics and dissemination No ethical approval is needed, and the results of this review will be disseminated via peer-reviewed publications and conference presentations. Trial registration number CRD42019128285.

\section{INTRODUCTION}

Stroke is one of the main causes of longterm disabilities among adults. ${ }^{1}$ Up to $85 \%$
Strengths and limitations of this study

To the best of our knowledge, this is the first systematic review for the effectiveness of interventions to improve hand motor function in individuals with moderate to severe stroke.

- The results of this systematic review will provide a detailed summary of the current progress of evidence for interventions to improve hand motor function, which will contribute to offering valuable information for therapeutists to help stroke survivors with moderate to severe impairment and identifying the gaps in the literature for further research.

- We anticipate that a limited meta-analysis is likely to be conducted because there may be significant heterogeneity owing to wide range of outcome measures, types of intervention and duration and frequency of training.

- There is always a possibility that the review does not identify all evidence or limitations relevant to the research question, such as the introduction of language bias due to the selection of studies published only in English.

of stroke survivors have hemiparesis that affects the upper extremity on one side, ${ }^{2}$ and less than half of them can regain proper arm function 6 months after stroke. ${ }^{3}$ Generally, hemiparesis impacts the movement function of the hand and wrist more than shoulder and elbow. ${ }^{35}$ As we know, hand movement plays a core role in upper limb function because of its indispensable and sophisticated function in human daily lives. ${ }^{6}$ Many vital activities of daily living, such as using a fork, buttoning a shirt, and opening a door handle, require various hand functions. ${ }^{7}$ The losses in hand function can seriously affect patients' functional independence and quality of life. ${ }^{8}$

Currently, for mildly impaired stroke survivors (about 20\%-25\%), ${ }^{9}$ constraint-induced movement therapy (CIMT) has been reported to produce significantly greater gains in hand/arm function compared with 
conventional therapy. ${ }^{10}{ }^{11}$ However, for stroke survivors who have moderate to severe impairment and do not meet the inclusion criteria of CIMT, ${ }^{12}{ }^{13}$ intervention options for hand motor function recovery are limited. The complexity of hand structure and function together with the neural basis that supports hand function might contribute to the great difficulty of hand function rehabilitation after stroke. Enormous biomechanical complexity makes the hand extensively represented in a large region of the motor cortex of the brain, ${ }^{14}$ which suggests that fine control of hand movement depends heavily on an intact corticospinal tract. When the ipsilesional corticofugal tract is serious damaged due to stroke, contralesional motor-related cortical recruitment becomes the main neural compensatory model for these moderate to severe stroke patients, according to previous studies. ${ }^{15}$ That the inhibition of contralesional motor cortex using transcranial magnetic stimulation ${ }^{16}$ or transcranial direct current stimulation (tDCS) ${ }^{17}$ can lead to more disrupted performance of a simple motor task in patients with poorer motor outcome serves as evidence to support such opinion. The contralesional cortical recruitment may rely on contralesional corticobulbospinal tract such as the corticoreticulospinal tract to control the affected upper limb. ${ }^{18}$ However, the compensatory corticoreticulospinal tract branches at multiple segments in spinal cord, and innervates proximal muscles more than distal ones, and prefers the flexors but lacks comparable resolution and innervation to hand and finger extensor muscles. ${ }^{1920}$ The aforementioned features result in the abnormal involuntary coupling between shoulder abduction and wrist/finger flexion, which is also known as the 'flexion synergy', as well as muscle weakness especially at extensors of distal joints, thus further constrains functional hand movements especially hand opening. ${ }^{21}{ }^{22}$ In short, it seems that extension at distal joints, like hand opening, depends more on the function of corticospinal track, primarily projected from the lesioned hemisphere, and lacks compensatory neural system to provide 'backup' driving. This neural basis makes effective restoration of hand function in moderate to severe stroke patients become extremely challenging. Furthermore, the resulting 'none-use-decay' can cause further decrease of the hand function. Although full of challenges, some of the research findings demonstrate that hand function recovery in this population is still feasible, with evidence showing both feasibility in intervention-induced changes in behaviour ${ }^{23}{ }^{24}$ and neural plasticity measures. ${ }^{20}$ We therefore focus on hand function recovery in the group of stroke survivors with moderate to severe impairment in this systematic review.

According to our knowledge of the literature, ample summary of the efficacy of various interventions for upper limb function rehabilitation in stroke patients can be found in published systematic reviews. Most of these reviews evaluate the efficacy of a single category of therapeutic technique, such as CIMT, ${ }^{25-29}$ robot-assisted therapy, ${ }^{30-35}$ bilateral training, ${ }^{36}{ }^{37}$ task-oriented training ${ }^{38}$ exercise therapy, ${ }^{39}$ functional electrical stimulation (FES) ${ }^{4041}$ orthotics, ${ }^{42} 43$ mental practice, ${ }^{4445}$ mirror therapy, ${ }^{46}{ }^{47}$ action observation, ${ }^{48}$ non-invasive cerebral stimulation, ${ }^{49-52}$ brain-computer interface, ${ }^{53} 54$ virtual reality, ${ }^{42}$ home-based therapy programmes ${ }^{55}$ and so on. There are also some comprehensive systematic reviews on general function treatment of upper limb after stroke $\mathrm{k}^{56-58}$ or other specific problems, such as motor dysfunction, ${ }^{59}{ }^{60}$ sensory impairment, ${ }^{61}$ spasticity, ${ }^{62} 63$ decreased quality of life, ${ }^{64}$ and shoulder pain and subluxation. ${ }^{65-67}$ In addition, other important issues of upper limb rehabilitation after stroke, like timing of intervention, ${ }^{68}$ dose of training, ${ }^{69}$ effects of severity on motor recovery, ${ }^{24}$ outcome measures ${ }^{70-75}$ and predictors of functional restoration ${ }^{76}$ were systematically reviewed as well. However, much less attention has been paid to the systematic review of hand function rehabilitation after moderate to severe stroke. ${ }^{78-83}$ Fortunately, with growing attention to this research field in recent decades, an increased number of clinical trials that focus on moderate to severe stroke patients is now available, involving various intervention methods, like electromyography (EMG)-triggered electrical stimulation, ${ }^{84}$ tDCS, ${ }^{85}$ robot-assisted movement training, repetitive transcranial magnetic stimulation ${ }^{86}$ and mirror therapy. ${ }^{87}$ Although with relatively small sample sizes, a review of these reported works will provide insight for the future direction along this line of research and thus may further impact future clinical practice for this large population.

Comprehensive overview of hand motor function rehabilitation in individuals with moderate to severe stroke has long been neglected not only in systematic reviews but also in the main guidelines for stroke rehabilitation. In the most recent Guidelines for Adult Stroke Rehabilitation and Recovery released by the American Heart Association and the American Stroke Association in 2016, we can only locate recommendations for the treatment of upper extremity activity but can hardly find any evidence-based suggestions for hand function training. ${ }^{88}$ The 2015 update of the Canadian Stroke Best Practice Recommendations: Stroke Rehabilitation Practice Guidelines has provided a series of recommendations on the management of upper extremity following stroke, including the restoration of sensorimotor function, and relief of spasticity and pain. Regarding the hand function rehabilitation after stroke, limited recommendations are scattered among evidence for other forms of upper extremity interventions, such as FES and CIMT for hand motor function, botulinum toxin for hand spasticity and range of motion, and exercise and massage for hand oedema ${ }^{89}$ Similar problems can be found in stroke rehabilitation guidelines in UK and Australia, which mainly provide recommendations on upper extremity management while lacking a detailed description of the current evidence on hand function recovery. ${ }^{90}{ }^{91}$ The absence of systematic evidence in guidelines for hand rehabilitation following stroke greatly increases the difficulty of clinical work. 
In short, a standardised systematic review on the effectiveness of interventions is warranted to improve hand motor function in individuals with moderate to severe stroke. Therefore, the aim of this review is to provide an overview of the following:

1. To identify which interventions that have been employed to increase hand function in individuals with moderate to severe stroke.

2. To verify the effectiveness of these interventions.

3. To identify the gaps in the literature.

\section{METHODS AND ANALYSES \\ Study design}

The review protocol was written and reported following the Preferred Reporting Items for Systematic Reviews and Meta-Analysis Protocols (PRISMA-P) (see the Preferred Reporting Items for Systematic Reviews and Meta-Analyses (PRISMA-P) checklist in online supplementary table 1) ${ }^{92}{ }^{93}$ For the results of this systematic review, we will publish it following the PRISMA statement. ${ }^{94} 95$

\section{Eligibility criteria}

Types of study

We will include all randomised controlled trials (RCTs) published in English that investigated the efficacy of rehabilitation interventions to improve hand motor function in individuals with moderate to severe stroke. The random allocation process should be performed in a standard way. Quasi-RCTs or trials without control group such as case series and case reports will be excluded. Preliminary and pilot studies, abstracts published in congress and conferences will also be excluded.

\section{Participants}

We will include all RCTs which have recruited adult patients ( $\geq 18$ years of age) with first or recurrent stroke. Stroke is defined as a clinical syndrome consisting of rapidly developing clinical signs of focal (or global in case of coma) disturbance of cerebral function lasting more than 24 hours or leading to death with no apparent cause other than a vascular origin' by WHO. ${ }^{96}$ The diagnosis of stroke should be confirmed by CT or MRI. The participants in all trials should be assessed as moderate to severe unilateral hand dysfunction as indicated by hand functional assessments such as the Fugl-Meyer Upper Extremity Scale $(<45)$ and Chedoke-McMaster Stroke Assessment (Sstage 4). ${ }^{97} 98$ Patients with subarachnoid haemorrhage or subdural haematoma will be excluded. Studies with participants with transient ischaemic attack will be excluded since all neurological symptoms would disappear.

\section{Types of interventions}

We will select all trials assessing interventions that at least have one of the treatment goals targeting the regaining of post-hand hand function in individuals with moderate to severe stroke. These interventions should be compared with a control intervention (eg, no treatment, standard care, conventional training or the same intervention method with different parameters). Trials focusing only on the training of elbow and shoulder will be excluded. The interventions here encompass many different, individual interventions, such as FES, mirror therapy, robot training, CIMT, brain-computer interface, repetitive transcranial magnetic stimulation and so on. Interventions can either be one-to-one or in group setting, hospital-based or home-based (under the supervision of professional), supervised by therapists or self-training. No limits will be placed on the timing, frequency and duration of interventions.

\section{Type of outcome measures}

The primary outcomes of this systematic review will focus on changes in patients' hand function using various assessments from baseline to the last available follow-up. The assessments can be divided into two groups ${ }^{72} 74$ : body functions measures (targeting impairments of hand function, such as Fugl-Meyer Assessment hand part, Chedoke-McMaster Stroke Assessment, Motricity Index, etc) and activity measures (assessing limitations of activities, such as Action Research Arm Test, Box and Block Test, Wolf Motor Function Test, etc).

Secondary outcome measures will include kinematic analysis of hand movement, possible improvements of quality of life, and mental health improvements related to the hand motor function recovery. The adverse events associated with interventions and adherence to treatment will also be considered.

\section{Search strategy for the identification of relevant studies}

Electronic searches will be performed for potentially eligible RCTs in the PubMed, CINAHL, Physiotherapy Evidence Database and Cochrane Library databases with restriction in articles with full texts in English. The date range parameters used in all databases will be between January 1999 and January 2019. Searches will combine terms from medical subject headings and keywords in title, abstract and text for the population, intervention and outcomes. The Cochrane Library Database search strategy in table 1 will be adapted for other databases. Furthermore, RCTs will also be obtained from the reference lists of included studies and published systematic reviews of interventions to improve upper limb or hand motor function in individuals with moderate to severe stroke.

\section{Screening of the studies}

The reference management software, Endnote (V.X9; Thomson Reuters, New York, USA), will be used to help upload, store and select the literature results. For each database, a separate library group will be created to keep all original search results. All separate library group copies will then merge into a new library group and duplicate checking will be carried out in the new library group using a Find Duplicates dialogue box in the Endnote. Two independent reviewers (HWW, RA) will screen all 


\begin{tabular}{|c|c|}
\hline$\# 1$ & MeSH descriptor: [Stroke] explode all trees \\
\hline \#2 & $\begin{array}{l}\text { Stroke:ti OR Cerebrovascular Accident*:ti OR CVA:ti OR Cerebrovascular Event*:ti OR Cerebrovascular Insult:ti } \\
\text { OR Brain:ti Vascular Accident*:ti OR Apoplexy*:ti OR Brain Infraction*:ti }\end{array}$ \\
\hline \#3 & \#1 OR \#2 \\
\hline \#4 & MeSH descriptor: [Hand] explode all trees \\
\hline \#7 & MeSH descriptor: [Rehabilitation] explode all trees \\
\hline \#8 & MeSH descriptor: [Exercise] explode all trees \\
\hline \#9 & MeSH descriptor: [Therapeutics] explode all trees \\
\hline \#10 & (Rehab* OR Exercis* OR Therap* OR Treat*):ti,ab,kw \\
\hline \#12 & \#7 OR \#8 OR \#9 OR \#10 OR \#11 \\
\hline \#13 & \#3 AND \#6 AND \#12 in Trials \\
\hline
\end{tabular}

$\mathrm{MeSH}$, medical subject headings.

the retrieved titles and abstracts according to the previously determined inclusion and exclusion criteria and full text will be screened to further confirm the final selection of the publications. Additional articles might be included by reference list check of the selected studies and relevant published systematic reviews mentioned in search strategy. In case of any disagreements, a third reviewer (JY) will be referred to make a final decision. All reasons for exclusion of any publications will be noted. The PRISMA flow of information through the different phases of a systematic review will be filled in, to record the whole screening process in detail. ${ }^{9495}$

\section{Data extraction}

Two independent reviewers (HWW, RA) will carry out the data extraction following recommendations from the PRISMA statement. ${ }^{94}{ }^{95}$ Disagrees between the two reviewers will be solved by a third reviewer (JY) to reach a consensus. The extracted data will include general study information (authors, year of publication and ethics), characteristics of participants (sample size, inclusion/ exclusion criteria, random process and allocation, age, gender, type and time since the onset of the stroke), interventions (type of intervention, dose, duration, frequency, supervision and comparison/control group), outcome measures (observation time points, hand function assessments, hand movement kinematic analysis, quality of life changes, possible mental improvement, dropout, length of follow-up, adverse events and conflict of interest). If necessary, the corresponding authors of the selected publications will be contacted for missing data and further information.

\section{Risk of bias}

The risk of bias of the included RCTs will be evaluated by the Cochrane Collaboration's tool (table 8.5.a in the Cochrane Handbook for Systematic Reviews of Interventions).$^{99}$ The Cochrane Collaboration's tool is a six-item checklist, which includes sequence generation, allocation concealment, blinding, incomplete outcome data, selective outcome reporting and other sources of bias not issued in other domains mentioned above. For each item in the checklist, the risk of bias will be categorised as low (meet all criteria), unclear (insufficient detail reported in the publications) or high risk of bias (meet none of the criteria). Two independent reviewers (HWW, RA) will perform these judgements of risk of bias and disagreements will be resolved first by discussion and then by referring to a third reviewer author (JY) as an arbitrator when necessary.

\section{Strategy for data synthesis}

We will provide a qualitative synthesis, in text and table, to summarise the main results of the selected publications. A narrative synthesis will be included to demonstrate the findings, structured around the type of intervention, target population characteristics, intervention content and types of outcome. We will check the heterogeneity of included studies by performing the $\chi^{2}$ test (significant level: 0.1 ) and the $\mathrm{I}^{2}$ statistic (high levels of heterogeneity: $\left.I^{2} \geq 50 \%\right)$. For studies that have sufficient data, and are homogeneous regarding the interventions and outcome measures, we will synthesise the results in meta-analysis using the Review Manager software (RevMan, V.5.3). In 
Table 2 Quality of evidence and definitions

$\begin{array}{ll}\text { High quality } & \text { Further research is very unlikely to change the confidence in the estimate of effects } \\ \text { Moderate quality } & \begin{array}{l}\text { Further research is likely to have an important impact on the confidence in the estimate of } \\ \text { effect and may change the estimate }\end{array} \\ \text { Low quality } & \begin{array}{l}\text { Further research is very likely to have an important impact on the confidence in the effect } \\ \text { and is likely to change the estimate }\end{array} \\ \text { Very low quality } & \text { Any estimate of the effect is very uncertain }\end{array}$

case of substantial heterogeneity, only qualitative synthesis will be performed.

\section{Analyses of subgroups or subsets}

We will perform the subgroups analyses if sufficient data are available. These analyses will involve differences between the stroke phases (ie, acute/subacute/chronic), the main therapeutic goal of treatment (ie, aiming at the recovery of hand function/aiming at the recovery of arm and hand function), the measurement tools (eg, activity measures/body function measures), intervention details (type, duration and delivery of the intervention), participation of patients in trials (active movement training/ passive training) and quality and risk of bias.

\section{Quality of evidence}

According to the recommendations from the Cochrane Handbook for Systematic Reviews of Interventions, ${ }^{99}$ the Grading of Recommendations Assessment, Development and Evaluation system will be used to assess the body of the evidence for all outcomes. ${ }^{100}$ This system involves consideration of within-study risk of bias, consistency, directness of evidence, precision of effects estimates and publication bias. The overall quality of evidence will be adjudicated at four levels: high, moderate, low and very low (table 2).

\section{Ethics and dissemination}

This systematic review does not need ethical approval and informed consent. Findings of this review will be disseminated via peer-reviewed publications and conference presentations.

\section{Patient and public involvement}

No patient involved.

\section{DISCUSSION}

Rehabilitation of hand motor function after stroke is different from other parts of the body like the lower extremity, trunk and even the proximal part of the upper limb, which recover faster and more completely. ${ }^{101}$ The neural basis underlying the hand rehabilitation in moderate to severe stroke patients makes effective restoration of hand motor function extremely challenging, therefore, currently this cohort of stroke survivors is largely ignored for hand function rehabilitation. To date, there is also no systematic review or guideline that focuses extensively on the effectiveness of interventions to improve hand motor function in individuals with moderate to severe stroke. To the best our knowledge, this is the first systematic review that concentrates on hand rehabilitation approaches in moderate to severe stroke patients and attempts to make a comprehensive analysis of the existing evidence to fill in the gaps in this research field.

This systematic review has several strengths. First, the preparation of this protocol is consistent with the methodology recommended by the PRISMA-P and the Cochrane handbook for systematic reviews of interventions. Second, we only include RCTs which have recruited participants with moderate to severe hand function after stroke. This is because publications have provided us with convincing evidence that patients with baseline ability to control wrist and finger extension can achieve improvements in hand function and quality of life after receiving treatment procedures like modified CIMT. ${ }^{12}{ }^{13}$ However, there is no consensus on the effectiveness of intervention methods for stroke patients with more severely impaired hand function. Third, more and more clinical trials on this topic have been published in recent decades, and the time for a systematic review is now.

The results of this systematic review will provide a detailed summary of the current progress of evidence for interventions to improve hand motor function in individuals with moderate to severe stroke. Such a review can contribute by not only identifying the gaps, thus providing guidance for further research, but by also offering valuable information for therapeutics to help stroke survivors with impaired hand function.

Contributors JY is the lead and the guarantor of this review. HW and RA conceptualised the review and drafted the manuscript. HW and RA developed the search strategy included in the protocol. JJ, LD and SC revised the protocol critically. All authors read and provided feedback on the draft and approved the final manuscript.

Funding This research is funded by National Key R\&D Program of China (Grant No. 2018YFC2002300 and 2018YFC2002301), the China National Nature Science Young Foundation (Grant No. 81401859) and the Science and Technology Commission of Shanghai Municipality (Grant Nos. 15441901602 and 16441905303).

Competing interests None declared.

Patient consent for publication Not required.

Provenance and peer review Not commissioned; externally peer reviewed. 
Open access This is an open access article distributed in accordance with the Creative Commons Attribution Non Commercial (CC BY-NC 4.0) license, which permits others to distribute, remix, adapt, build upon this work non-commercially, and license their derivative works on different terms, provided the original work is properly cited, appropriate credit is given, any changes made indicated, and the use is non-commercial. See: http://creativecommons.org/licenses/by-nc/4.0/.

\section{ORCID iDs}

Hewei Wang http://orcid.org/0000-0001-7632-0607

Jie Jia http://orcid.org/0000-0002-4516-4629

\section{REFERENCES}

1. Feigin VL, Krishnamurthi RV, Parmar P, et al. Update on the global burden of ischemic and hemorrhagic stroke in 1990-2013: the GBD 2013 study. Neuroepidemiology 2015;45:161-76.

2. Mayo NE, Wood-Dauphinee S, Ahmed S, et al. Disablement following stroke. Disabil Rehabil 1999;21:258-68.

3. Kwakkel G, Kollen BJ, van der Grond J, et al. Probability of regaining dexterity in the flaccid upper limb: impact of severity of paresis and time since onset in acute stroke. Stroke 2003:34:2181-6.

4. Lee KB, Lim SH, Kim KH, et al. Six-Month functional recovery of stroke patients: a multi-time-point study. Int $J$ Rehabil Res 2015;38:173-80.

5. Ferraro M, Demaio JH, Krol J, et al. Assessing the motor status score: a scale for the evaluation of upper limb motor outcomes in patients after stroke. Neurorehabil Neural Repair 2002;16:283-9.

6. Neumann DA. Kinesiology of the musculoskeletal system-e-book: foundations for rehabilitation. Elsevier Health Sciences, 2013.

7. Wolbrecht ET, Rowe JB, Chan V, et al. Finger strength, individuation, and their interaction: relationship to hand function and corticospinal tract injury after stroke. Clin Neurophysiol 2018;129:797-808.

8. Hung C-S, Hsieh Y-W, Wu C-Y, et al. The effects of combination of robot-assisted therapy with task-specific or Impairment-Oriented training on motor function and quality of life in chronic stroke. $P m R$ 2016;8:721-9.

9. Wolf SL, Blanton S, Baer H, et al. Repetitive task practice: a critical review of constraint-induced movement therapy in stroke. Neurologist 2002;8:325-38.

10. Wolf SL, Winstein CJ, Miller JP, et al. Effect of constraintinduced movement therapy on upper extremity function 3 to 9 months after stroke: the excite randomized clinical trial. JAMA 2006;296:2095-104.

11. Taub E, Uswatte G, Elbert T. New treatments in neurorehabiliation founded on basic research. Nat Rev Neurosci 2002;3:228-36.

12. Kwakkel G, Veerbeek JM, van Wegen EEH, et al. Constraintinduced movement therapy after stroke. Lancet Neurol 2015:14:224-34.

13. Morris Det al. Constraint-induced movement therapy for moter recovery after stroke. NeuroRehabilitation 1997:9:29-43.

14. Hlustík P, Solodkin A, Gullapalli RP, et al. Somatotopy in human primary motor and somatosensory hand representations revisited. Cereb Cortex 2001;11:312-21.

15. Hamzei F, Dettmers C, Rijntjes M, et al. The effect of cortico-spinal tract damage on primary sensorimotor cortex activation after rehabilitation therapy. Exp Brain Res 2008;190:329-36.

16. Johansen-Berg H, Rushworth MFS, Bogdanovic MD, et al. The role of ipsilateral premotor cortex in hand movement after stroke. Proc Natl Acad Sci U S A 2002;99:14518-23.

17. Yao J, Drogos J, Veltink F, et al. The effect of transcranial direct current stimulation on the expression of the flexor synergy in the paretic arm in chronic stroke is dependent on shoulder abduction loading. Front Hum Neurosci 2015;9:262.

18. Baker SN, Zaaimi B, Fisher KM, et al. Pathways mediating functional recovery. Prog Brain Res 2015;218:389-412.

19. Lawrence DG, Kuypers HG. The functional organization of the motor system in the monkey. I. The effects of bilateral pyramida lesions. Brain : a journal of neurology 1968;91:1-14.

20. Wilkins $\mathrm{KB}$, Owen $\mathrm{M}$, Ingo $\mathrm{C}$, et al. Neural plasticity in moderate to severe chronic stroke following a Device-Assisted task-specific Arm/Hand intervention. Front Neurol 2017;8.

21. Lan Y, Yao J, Dewald JPA. The impact of shoulder abduction loading on volitional hand opening and grasping in chronic hemiparetic stroke. Neurorehabil Neural Repair 2017;31:521-9.

22. Lang CE, Schieber MH. Reduced muscle selectivity during individuated finger movements in humans after damage to the motor cortex or corticospinal tract. J Neurophysiol 2004;91:1722-33.
23. Camona C, Wilkins KB, Drogos J, et al. Improving hand function of severely impaired chronic hemiparetic stroke individuals using taskspecific training with the Reln-Hand system: a case series. Front Neurol 2018;9.

24. Hayward K, Barker R, Brauer S. Interventions to promote upper limb recovery in stroke survivors with severe paresis: a systematic review. Disabil Rehabil 2010;32:1973-86.

25. Corbetta D, Sirtori V, Castellini G, et al. Constraint-induced movement therapy for upper extremities in people with stroke. Cochrane Database Syst Rev 2015;18.

26. Etoom M, Hawamdeh M, Hawamdeh Z, et al. Constraint-induced movement therapy as a rehabilitation intervention for upper extremity in stroke patients: systematic review and meta-analysis. Int J Rehabil Res 2016;39:197-210.

27. McIntyre A, Viana R, Janzen S, et al. Systematic review and metaanalysis of constraint-induced movement therapy in the hemiparetic upper extremity more than six months post stroke. Top Stroke Rehabil 2012;19:499-513.

28. Shi YX, Tian JH, Yang KH, et al. Modified constraint-induced movement therapy versus traditional rehabilitation in patients with upper-extremity dysfunction after stroke: a systematic review and meta-analysis. Arch Phys Med Rehabil 2011;92:972-82.

29. Nijland R, Kwakkel G, Bakers J, et al. Constraint-induced movement therapy for the upper paretic limb in acute or sub-acute stroke: a systematic review. Int J Stroke 2011;6:425-33.

30. Veerbeek JM, Langbroek-Amersfoort AC, van Wegen EEH, et al. Effects of robot-assisted therapy for the upper limb after stroke. Neurorehabil Neural Repair 2017:31:107-21.

31. Zhang C, Li-Tsang CWP, Au RKC. Robotic approaches for the rehabilitation of upper limb recovery after stroke: a systematic review and meta-analysis. Int J Rehabil Res 2017;40:19-28.

32. Bertani R, Melegari C, De Cola MC, et al. Effects of robot-assisted upper limb rehabilitation in stroke patients: a systematic review with meta-analysis. Neurol Sci 2017;38:1561-9.

33. Babaiasl M, Mahdioun SH, Jaryani P, et al. A review of technological and clinical aspects of robot-aided rehabilitation of upper-extremity after stroke. Disabil Rehabil Assist Technol 2016;11:263-80.

34. Prange GB, Jannink MJA, Groothuis-Oudshoorn CGM, et al Systematic review of the effect of robot-aided therapy on recovery of the hemiparetic arm after stroke. JRRD 2006;43:171-84.

35. Mehrholz J, Platz T, Kugler J, et al. Electromechanical and robotassisted arm training for improving arm function and activities of daily living after stroke. Cochrane Database Syst Rev 2008;4.

36. Coupar F, Pollock A, van Wijck F, et al. Simultaneous bilateral training for improving arm function after stroke. Cochrane Database Syst Rev 2010;33.

37. Latimer CP, Keeling J, Lin B, et al. The impact of bilateral therapy on upper limb function after chronic stroke: a systematic review. Disabil Rehabil 2010;32:1221-31.

38. Timmermans AAA, Spooren AIF, Kingma $\mathrm{H}$, et al. Influence of taskoriented training content on skilled arm-hand performance in stroke: a systematic review. Neurorehabil Neural Repair 2010;24:858-70.

39. van der Lee JH, Snels IAK, Beckerman H, et al. Exercise therapy for arm function in stroke patients: a systematic review of randomized controlled trials. Clin Rehabil 2001;15:20-31.

40. Eraifej J, Clark W, France B, et al. Effectiveness of upper limb functional electrical stimulation after stroke for the improvement of activities of daily living and motor function: a systematic review and meta-analysis. Syst Rev 2017;6:40.

41. Vafadar AK, Côté JN, Archambault PS. Effectiveness of functiona electrical stimulation in improving clinical outcomes in the upper arm following stroke: a systematic review and meta-analysis. Biomed Res Int 2015;2015:1-14.

42. Tyson SF, Kent RM. The effect of upper limb orthotics after stroke: a systematic review. NeuroRehabilitation 2011;28:29-36.

43. Lannin NA, Novak I, Cusick A. A systematic review of upper extremity casting for children and adults with central nervous system motor disorders. Clin Rehabil 2007;21:963-76.

44. Barclay-Goddard RE, Stevenson TJ, Poluha W, et al. Mental practice for treating upper extremity deficits in individuals with hemiparesis after stroke. Cochrane Database Syst Rev 2011;5.

45. Guerra ZF, Lucchetti ALG, Lucchetti G. Motor imagery training after stroke: a systematic review and meta-analysis of randomized controlled trials. J Neurol Phys Ther 2017;41:205-14.

46. Ezendam D, Bongers RM, Jannink MJA. Systematic review of the effectiveness of mirror therapy in upper extremity function. Disabil Rehabil 2009;31:2135-49.

47. Pérez-Cruzado D, Merchán-Baeza JA, González-Sánchez M, et al. Systematic review of mirror therapy compared with conventional rehabilitation in upper extremity function in stroke survivors. Aust Occup Ther J 2017;64:91-112. 
48. Thieme H, Morkisch N, Mehrholz J, et al. Mirror therapy for improving motor function after stroke. Cochrane Database Syst Rev $2018 ; 17$

49. Elsner B, Kugler J, Pohl M, et al. Transcranial direct current stimulation (tDCS) for improving function and activities of daily living in patients after stroke. The Cochrane database of systematic reviews 2013;11.

50. Kandel M, Beis J-M, Le Chapelain L, et al. Non-Invasive cerebral stimulation for the upper limb rehabilitation after stroke: a review. Ann Phys Rehabil Med 2012;55:657-80.

51. Graef P, Dadalt MLR, Rodrigués DAMdaS, et al. Transcranial magnetic stimulation combined with upper-limb training for improving function after stroke: a systematic review and metaanalysis. J Neurol Sci 2016;369:149-58.

52. Tedesco Triccas L, Burridge JH, Hughes AM, et al. Multiple sessions of transcranial direct current stimulation and upper extremity rehabilitation in stroke: a review and meta-analysis. Clinical Neurophysiology 2016;127:946-55.

53. Monge-Pereira E, Ibañez-Pereda J, Alguacil-Diego IM, et al. Use of electroencephalography brain-computer interface systems as a rehabilitative approach for upper limb function after a stroke: a systematic review. PM\&R 2017;9:918-32.

54. Carvalho R, Dias N, Cerqueira JJ. Brain-machine interface of upper limb recovery in stroke patients rehabilitation: a systematic review. Physiotherapy Research International 2019;24:e1764.

55. Coupar F, Pollock A, Legg LA, et al. Home-Based therapy programmes for upper limb functional recovery following stroke. Cochrane Database of Systematic Reviews 2012;29.

56. Pollock A, Farmer SE, Brady MC, et al. Interventions for improving upper limb function after stroke. Cochrane Database Syst Rev 2014;23.

57. Urton ML, Kohia M, Davis J, et al. Systematic literature review of treatment interventions for upper extremity hemiparesis following stroke. Occup Ther Int 2007;14:11-27.

58. Platz T. Evidence-based arm rehabilitation-a systematic review of the literature]. Der Nervenarzt 2003;74:841-9.

59. Hatem SM, Saussez G, Della Faille M, et al. Rehabilitation of motor function after stroke: a multiple systematic review focused on techniques to stimulate upper extremity recovery. Front Hum Neurosci 2016;10:442.

60. Pelton $\mathrm{T}$, van Vliet $\mathrm{P}$, Hollands $\mathrm{K}$. Interventions for improving coordination of reach to GRASP following stroke: a systematic review. Int J Evid Based Healthc 2012;10:89-102.

61. Doyle S, Bennett S, Fasoli SE, et al. Interventions for sensory impairment in the upper limb after stroke. Cochrane Database of Systematic Reviews 2010;51(4-e Suppl).

62. Dong Y, Wu T, Hu X, et al. Efficacy and safety of botulinum toxin type A for upper limb spasticity after stroke or traumatic brain injury: a systematic review with meta-analysis and trial sequential analysis. Eur J Phys Rehabil Med 2017;53:256-67.

63. Salazar AP, Pinto C, Ruschel Mossi JV, et al. Effectiveness of static stretching positioning on post-stroke upper-limb spasticity and mobility: systematic review with meta-analysis. Ann Phys Rehabil Med 2018.

64. Pulman J, Buckley E. Assessing the efficacy of different upper limb hemiparesis interventions on improving health-related quality of life in stroke patients: a systematic review. Top Stroke Rehabil 2013;20:171-88.

65. Arya KN, Pandian S, Puri V. Rehabilitation methods for reducing shoulder subluxation in post-stroke hemiparesis: a systematic review. Top Stroke Rehabil 2018;25:68-81.

66. Lee S-H, Lim SM. Acupuncture for poststroke shoulder pain: a systematic review and meta-analysis. Evidence-Based Comp Alternative Med 2016;2016:1-8.

67. Nadler M, Pauls MMH. Shoulder orthoses for the prevention and reduction of hemiplegic shoulder pain and subluxation: systematic review. Clin Rehabil 2017;31:444-53.

68. Wattchow KA, McDonnell MN, Hillier SL. Rehabilitation interventions for upper limb function in the first four weeks following stroke: a systematic review and meta-analysis of the evidence. Arch Phys Med Rehabil 2018;99:367-82.

69. Hayward KS, Brauer SG. Dose of arm activity training during acute and subacute rehabilitation post stroke: a systematic review of the literature. Clin Rehabil 2015;29:1234-43.

70. Ashford S, Slade M, Malaprade F, et al. Evaluation of functional outcome measures for the hemiparetic upper limb: a systematic review. J Rehabil Med 2008;40:787-95.

71. Beaulieu L-D, Milot M-H. Changes in transcranial magnetic stimulation outcome measures in response to upper-limb physical training in stroke: a systematic review of randomized controlled trials. Ann Phys Rehabil Med 2018;61:224-34.
72. Alt Murphy M, Resteghini C, Feys P, et al. An overview of systematic reviews on upper extremity outcome measures after stroke. $B M C$ Neurol 2015;15:29

73. Sivan M, O'Connor RJ, Makower S, et al. Systematic review of outcome measures used in the evaluation of robot-assisted upper limb exercise in stroke. J Rehabil Med 2011;43:181-9.

74. Santisteban L, Térémetz M, Bleton J-P, et al. Upper limb outcome measures used in stroke rehabilitation studies: a systematic literature review. PLoS One 2016;11:e0154792.

75. Velstra I-M, Ballert CS, Cieza A. A systematic literature review of outcome measures for upper extremity function using the International classification of functioning, disability, and health as reference. $P m R$ 2011;3:846-60.

76. Kumar P, Kathuria P, Nair P, et al. Prediction of upper limb motor recovery after subacute ischemic stroke using diffusion tensor imaging: a systematic review and meta-analysis. J Stroke 2016;18:50-9.

77. Tedesco Triccas L, Kennedy N, Smith T, et al. Predictors of upper limb spasticity after stroke? A systematic review and meta-analysis. Physiotherapy 2019;105:163-73.

78. Ostolaza M, Abudarham J, Dilascio S, et al. Hand fine motor skills and use of both hand and arm in subjects after a stroke: a systematic review]. Revista de neurologia 2017;64:289-98.

79. Grant VM, Gibson A, Shields N. Somatosensory stimulation to improve hand and upper limb function after stroke-a systematic review with meta-analyses. Top Stroke Rehabil 2018;25:150-60.

80. Yue Z, Zhang X, Wang J. Hand rehabilitation robotics on poststroke motor recovery. Behav Neurol 2017;2017:1-20.

81. Meilink A, Hemmen B, Seelen $\mathrm{H}$, et al. Impact of EMG-triggered neuromuscular stimulation of the wrist and finger extensors of the paretic hand after stroke: a systematic review of the literature. Clin Rehabil 2008;22:291-305.

82. McConnell AC, Moioli RC, Brasil FL, et al. Robotic devices and brain-machine interfaces for hand rehabilitation post-stroke. $J$ Rehabil Med 2017;49:449-60.

83. O'Brien AT, Bertolucci F, Torrealba-Acosta G, et al. Non-Invasive brain stimulation for fine motor improvement after stroke: a metaanalysis. Eur J Neurol 2018;25:1017-26.

84. Schick T, Schlake H-P, Kallusky J, et al. Synergy effects of combined multichannel EMG-triggered electrical stimulation and mirror therapy in subacute stroke patients with severe or very severe arm/hand paresis. Restor Neurol Neurosci 2017;35:319-32.

85. Rabadi $\mathrm{MH}$, Aston $\mathrm{CE}$. Effect of transcranial direct current stimulation on severely affected Arm-Hand motor function in patients after an acute ischemic stroke: a pilot randomized control trial. Am J Phys Med Rehabil 2017;96(10 Suppl 1):S178-84.

86. Takeuchi N, Tada T, Toshima M, et al. Inhibition of the unaffected motor cortex by $1 \mathrm{~Hz}$ repetitive transcranical magnetic stimulation enhances motor performance and training effect of the paretic hand in patients with chronic stroke. J Rehabil Med 2008;40:298-303.

87. Michielsen ME, Selles RW, van der Geest JN, et al. Motor recovery and cortical reorganization after mirror therapy in chronic stroke patients: a phase II randomized controlled trial. Neurorehab Neural Repair 2011;25:223-33.

88. Winstein CJ, Stein J, Arena R, et al. Guidelines for adult stroke rehabilitation and recovery: a guideline for healthcare professionals from the American heart Association/American stroke association. Stroke 2016;47:e98-169.

89. Hebert D, Lindsay MP, Mclntyre A, et al. Canadian stroke best practice recommendations: stroke rehabilitation practice guidelines, update 2015. Int J Stroke 2016;11:459-84.

90. Foundation S. Clinical guidelines for stroke management 2017. Victoria, Australia: Stroke Foundation Melbourne, 2017.

91. National Clinical Guideline C. National Institute for health and care excellence: clinical guidelines. stroke rehabilitation: long term rehabilitation after stroke. London: Royal College of Physicians (UK) National Clinical Guideline Centre, 2013.

92. Moher D, Shamseer L, Clarke M, et al. Preferred reporting items for systematic review and meta-analysis protocols (PRISMA-P) 2015 statement. Syst Rev 2015;4.

93. Shamseer L, Moher D, Clarke M, et al. Preferred reporting items for systematic review and meta-analysis protocols (PRISMA-P) 2015 elaboration and explanation. BMJ 2015;349:g7647.

94. Liberati A, Altman DG, Tetzlaff J, et al. The PRISMA statement for reporting systematic reviews and meta-analyses of studies that evaluate health care interventions: explanation and elaboration. $J$ Clin Epidemiol 2009;62:e1-34.

95. Moher D, Liberati A, Tetzlaff J, et al. Preferred reporting items for systematic reviews and meta-analyses: the PRISMA statement. PLoS Med 2009;6:e1000097-e97. 
96. Hatano S. Experience from a multicentre stroke register: a preliminary report. Bulletin of the World Health Organization 1976;54:541-53.

97. Hayward KS, Schmidt J, Lohse KR, et al. Are we armed with the right data? pooled individual data review of biomarkers in people with severe upper limb impairment after stroke. Neuroimage 2017;13:310-9.

98. Woytowicz EJ, Rietschel JC, Goodman RN, et al. Determining levels of upper extremity movement impairment by applying a cluster analysis to the Fugl-Meyer assessment of the upper extremity in chronic stroke. Arch Phys Med Rehabil 2017;98:456-62.

99. Higgins JP, Green S. Cochrane Handbook for systematic reviews of interventions, 2008

100. Guyatt GH, Oxman AD, Vist GE, et al. Grade: an emerging consensus on rating quality of evidence and strength of recommendations. BMJ 2008;336:924-6.

101. Duruöz MT. Hand function: a practical guide to assessment. Springer, 2014. 